OPEN ACCESS

Edited by:

Itamar Ronen,

Leiden University, Netherlands

Reviewed by:

Marco Palombo,

University College London,

United Kingdom

Gisela E. Hagberg,

Universität Tübingen, Germany

*Correspondence:

Sébastien Mériaux sebastien.meriaux@cea.fr

Specialty section:

This article was submitted to

Biomedical Physics,

a section of the journal

Frontiers in Physics

Received: 22 December 2017

Accepted: 13 April 2018

Published: 01 May 2018

Citation:

Mériaux S, Conti $A$ and Larrat $B$ (2018) Assessing Diffusion in the Extra-Cellular Space of Brain Tissue by Dynamic MRI Mapping of Contrast

Agent Concentrations.

Front. Phys. 6:38

doi: 10.3389/fphy.2018.00038

\section{Assessing Diffusion in the Extra-Cellular Space of Brain Tissue by Dynamic MRI Mapping of Contrast Agent Concentrations}

\author{
Sébastien Mériaux ${ }^{1,2 *}$, Allegra Conti ${ }^{1,2}$ and Benoît Larrat ${ }^{1,2}$ \\ ${ }^{1}$ NeuroSpin, Institut des Sciences du Vivant Frédéric-Joliot, Commissariat à l'Énergie Atomique et aux Énergies Alternatives, \\ Gif-sur-Yvette, France, ${ }^{2}$ Université Paris-Saclay, Orsay, France
}

The characterization of extracellular space (ECS) architecture represents valuable information for the understanding of transport mechanisms occurring in brain parenchyma. ECS tortuosity reflects the hindrance imposed by cell membranes to molecular diffusion. Numerous strategies have been proposed to measure the diffusion through ECS and to estimate its tortuosity. The first method implies the perfusion for several hours of a radiotracer which effective diffusion coefficient $D^{*}$ is determined after post mortem processing. The most well-established techniques are real-time iontophoresis that measures the concentration of a specific ion at known distance from its release point, and integrative optical imaging that relies on acquiring microscopy images of macromolecules labeled with fluorophore. After presenting these methods, we focus on a recent Magnetic Resonance Imaging (MRI)-based technique that consists in acquiring concentration maps of a contrast agent diffusing within ECS. Thanks to $\mathrm{MRI}$ properties, molecular diffusion and tortuosity can be estimated in 3D for deep brain regions. To further discuss the reliability of this technique, we point out the influence of the delivery method on the estimation of $D^{*}$. We compare the value of $D^{*}$ for a contrast agent intracerebrally injected, with its value when the agent is delivered to the brain after an ultrasound-induced blood-brain barrier (BBB) permeabilization. Several studies have already shown that tortuosity may be modified in pathological conditions. Therefore, we believe that MRI-based techniques could be useful in a clinical context for characterizing the diffusion properties of pathological ECS and thus predicting the drug biodistribution into the targeted area.

Keywords: brain tissue tortuosity, extracellular diffusion, MRI contrast agents, in vivo concentration quantification, dynamic $T_{1}$ mapping, ultrasound-induced BBB permeabilization

\section{WHY ASSESSING BRAIN TORTUOSITY?}

The diffusion of substances in the brain is predominantly occurring through the narrow extracellular space (ECS) that comprises the fluid-filled spaces external to cell membranes. A consequent number of studies (see [1] for an extensive review) have already established that the ECS labyrinthine nature makes the brain act like a porous medium for substances that cannot cross cellular membranes, allowing the use of established diffusion equation [2]. Two main structural 
descriptors, $\alpha$ and $\lambda$, are usually reported to define the diffusion processes in the ECS. The volume fraction $\alpha$ reflects the fact that molecules released into the ECS are restricted to a smaller volume than the entire brain volume. Furthermore, the diffusion of molecules can be considered as hindered by cells, because of the increase in path length imposed by the ECS geometry. This hindrance relatively to a free medium is quantified introducing the tissue tortuosity $\lambda[3]$ :

$$
\lambda=\sqrt{\frac{D_{\text {free }}}{D^{*}}}
$$

where $D_{\text {free }}$ is the diffusion coefficient in obstacle-free medium (water or very dilute gel), and $D^{*}$ the effective diffusion coefficient in ECS.

Volume fraction $\alpha$ admits of a simple interpretation and its value is reported to be $\sim 0.2$ for most regions of different studied brains [1]. Moreover, tortuosity $\lambda$, which value is around 1.6 for small molecules, remains a composite parameter as many potential mechanisms may contribute to the hindrance experienced by molecules. This parameter is commonly described as some combination of (i) an increased path length as molecules are compelled to diffuse around cellular obstructions [4, 5], (ii) a transient trapping in deadspace microdomains [6, 7], and (iii) extracellular matrix interactions $[8,9]$. When considering the ECS diffusion of larger macromolecules, such as dextrans or proteins, a new behavior occurs leading to an increase of $\lambda$ with the hydrodynamic diameter $\left(d_{H}\right)$. In this regime, the ECS porous structure is dominating and the observed restricted diffusion of molecules is mainly influenced by (i) the steric hindrance arising from the pore's limited cross-sectional area and (ii) the drag from the pore walls. Thorne and Nicholson performed tortuosity measurements using quantum dot nanocrystals $\left(d_{H} \sim 35 \mathrm{~nm}\right)$, and they were able to estimate that the true average ECS width in the in vivo rat cortex lies between 38 and $64 \mathrm{~nm}$ using specific pore models [10].

Although diffusion transport predominates in the ECS, it is often modified by loss of molecules through removal across the blood-brain barrier (BBB), uptake into cells, or binding to receptors. Furthermore, clearance processes may also be due to a hydrodynamic flow of fluid, but it seems likely that this bulk flow is confined to the perivascular spaces in healthy brain [11]. If the underlying mechanism, importance, and even the existence of interstitial fluid flow remain incompletely resolved, this question has become a topic of renewed interest with the introduction of the brain lymphatic drainage system, known as the glymphatic system [12-14]. This system, which involves convective transport from para-arterial to para-venous cerebrospinal fluid through ECS, has been proposed to account for solute clearance in brain, and for removing toxic metabolites from the brain [15]. However, Jin et al suggested that the glymphatic system flow is not essential,

Abbreviations: ECS, ExtraCellular Space; BBB, Blood-Brain Barrier; RTI, RealTime Iontophoresis; TMA, TetraMethylAmmonium; IOI, Integrative Optical Imaging; MRI, Magnetic Resonance Imaging; $\mathrm{ADC}_{\mathrm{W}}$, water Apparent Diffusion Coefficient; Gd, Gadolinium since the role of diffusion seems to remain dominant when observing the molecules dispersion in the ECS [16].

Thus, the precise determination of ECS diffusion properties represents valuable information for the understanding of brain physiology and drug delivery in normal or pathological conditions. To predict the distribution of a specific externally administered agent, it is essential to know its effective diffusion coefficient in brain tissue, as well as the relative importance of diffusion vs. clearance processes that may remove that agent from the ECS. For example, these parameters are crucial to control the dose-dependent action of pharmacological agents used to target specific brain diseases, and consequently to improve clinical treatment protocols.

\section{WHAT ARE THE WELL-ESTABLISHED TECHNIQUES FOR ASSESSING ECS DIFFUSION PROPERTIES?}

The concept underlying methods of diffusion measurements is to introduce a detectable substance into the ECS, to subsequently measure its concentration distribution in space and time. It is necessary for the measurement technique to produce concentration distribution curves rather than single values, so that the adequate diffusion equation can be applied to extract $\alpha$ and $\lambda$ values. The choice of diffusing probe is also key: it should be small enough to explore all ECS regions, but should also not cross cellular membranes or BBB to remain predominantly in the ECS compartment. Furthermore, the probe should be nontoxic to brain tissue and its concentration should be kept sufficiently low to avoid osmolarity modifications as well as sufficiently high to exceed the sensitivity threshold of the detection technique.

Lots of reviews have been written to detail the main drawbacks and advantages of each technique implemented to measure ECS diffusion properties, as well as to compare the $\alpha$ and $\lambda$ values obtained in various animal brains and physio-pathological conditions [1, 4, 17-19]. The first technique used radiolabeled probes, such as inulin, sulfate or dextran [20]. After several hours of probe perfusion in the ECS of anesthetized animal, its brain is removed, frozen and sectioned. The post mortem analysis of several brains processed at various times after perfusion allows deducing the $D^{*}$ value of radiotracer from the temporal evolution of radioactivity profile. The need of one animal for each time point is the main disadvantage explaining why this technique is not in general use today.

To overcome this drawback, Nicholson and Phillips proposed a "point-source paradigm," which consists in releasing specific small ions with a glass micropipette, and then measuring the resulting concentration $\sim 100 \mu \mathrm{m}$ away with an ion-selective microelectrodes (ISMs) [21]. This method is usually called RealTime Iontophoresis (RTI) because the source micropipette emits molecules using iontophoresis, thanks to the application of a constant current pulse. If the source amplitude is accurately defined, both $\alpha$ and $\lambda$ may be estimated. The ion employed for a vast majority of studies is the tetramethylammonium monovalent cation (TMA+). This molecule is broadly used because it does not affect physiological function at low concentrations, and it 
remains mostly extracellular for the duration of measurements. To date, RTI-TMA + remains the predominant technique for exploring ECS diffusion properties and their changes induced by brain development and aging, as well as numerous pathological states [1]. The major advantage of RTI method is the possibility to perform real-time measurements in very small volumes of living tissue, and therefore to follow diffusion properties in specific brain regions during drug injection or other intervention. The main drawbacks concern the relatively small number of usable probes, since each ion requires a dedicated ISM, and the concentration quantification that is only performed at a single position.

Nicholson and Tao recently introduced the Integrative Optical Imaging (IOI) technique, for quantifying in $2 \mathrm{D}$ the diffusion properties of larger probes such as proteins and macromolecules [22]. The method requires to label the probes with fluorophores, enabling their in vivo follow-up with dedicated epifluorescence microscope. The labeled probes are released in the ECS from a micropipette by a pressure pulse, and by fitting a 2D Gaussian curve to the image intensity at different time points, the $D^{*}$ value, and hence $\lambda$, can be estimated. The diffusion measurements performed in vivo with IOI technique exhibit at the same time an excellent sensitivity, a high spatial and temporal resolutions [10]. One main limitation is that optical detection can only be performed down to a depth of $\sim 400 \mu \mathrm{m}$ [18]. Still, the RTI and IOI methods are now well-established and present in most cases the same results in terms of estimated $D^{*}$ and $\lambda$ values $[1,17,18]$.

Water diffusion in the brain can be directly assessed with Magnetic Resonance Imaging (MRI) to probe tissue microstructure [23]. However, water is found in both intracellular and extracellular compartments, with specific exchange rates between them. The relationship between water movement, water apparent diffusion coefficient $\left(\mathrm{ADC}_{\mathrm{W}}\right)$ maps and changes in ECS characteristics thus remains difficult to understand since changes in both $\alpha$ and $\lambda$ are accompanied by changes in $\mathrm{ADC}_{\mathrm{w}}$ [24]. Nevertheless, diffusion MRI of protons from extracellular molecules such as TMA+ was recently demonstrated to be a potential alternative to the RTI method [25]. Another study investigated the use of 2FDG-6P as a compartment-specific marker in normal and globally ischemic rat brain, and followed its diffusion in ECS with dedicated ${ }^{19}$ F MRI strategy [26]. For these two studies, volume-localized diffusion spectroscopy sequences were chosen, leading to relatively poor spatial resolution.

In this Perspective article, we illustrate how a different MRI approach, which relies on the dynamic mapping of Gadolinium (Gd)-based contrast agent concentrations [27], could be complementary to RTI and IOI methods for studying the ECS diffusion properties in extended brain regions.

\section{OUR APPROACH TO ASSESS BRAIN TORTUOSITY WITH MRI}

In 2013, we introduced a different approach to assess brain tortuosity with MRI: a specific Gd-based contrast agent was delivered in the striatum of rat brains and its diffusion through the ECS was quantified thanks to the dynamic mapping of MRI probe concentrations using a dedicated $\mathrm{T}_{1}$ quantification strategy [27]. Interestingly, Hagberg et al. also relied on $\mathrm{T}_{1}$ mapping sequences to measure ECS diffusion properties from voxel-wise measurements of the temporal pharmacokinetic curve obtained after an intracerebral injection of Gd-based probe [28]. One difference between the two approaches is that ECS diffusion estimation comes from the spatial evolution of MRI signal in Marty et al. [27], rather than on the temporal one in Hagberg et al. [28]. Both studies succeeded in estimating $D^{*}$ for several contrast agents and providing brain tortuosity values, thanks to sensitive quantification strategies.

\section{MRI TORTUOSITY PROBE}

As already explained, the choice of MRI probe used for $\lambda$ measurements is key. We selected a clinically approved Gd-chelate named Dotarem ${ }^{\circledR}$ (Guerbet, France) that presents several advantages. It is nontoxic for brain tissue compared to other MRI contrast agents [29]. Its size $\left(d_{H}<1 \mathrm{~nm}\right)$ is close to the one of TMA $+\left(d_{H} \sim 0.6 \mathrm{~nm}\right)$ and small compared to the ECS typical width. Furthermore, Dotarem ${ }^{\circledR}$ is known to not cross cell membranes and thus remain into the extracellular compartment.

Finally, Dotarem ${ }^{\circledR}$ has a relatively good relaxivity $\left(r_{1}=\right.$ $3.4 \mathrm{mM}^{-1} \cdot \mathrm{s}^{-1}$ ) at high magnetic field (7T). This ensures to detect relatively low local concentrations (down to a few $\mu \mathrm{M}$ ), while maintaining sufficient spatial and temporal resolutions for detecting molecules spreading in the brain. Noticeably, Dotarem ${ }^{\circledR}$ is known to be a stable molecule that maintains its relaxivity unchanged in plasma and in several tissues [30].

\section{MRI QUANTIFICATION STRATEGY}

Quantitative techniques are requested to precisely map the distribution of MRI probe after its delivery to the brain and then estimate its $D^{*}$ value. Therefore, instead of relying on $\mathrm{T}_{1}$-weighted images which contrast may saturate at high Gd concentration, we implemented one $T_{1}$ mapping strategy based on the Inversion Recovery Fast Gradient Echo sequence presented in Figure 1A [31]. The sequence parameters were chosen to ensure a high sampling rate of the longitudinal magnetization recovery curve over a long time (Figures 1B,C): 60 images are acquired with inversion times between 45 and $5060 \mathrm{~ms}$ spaced by $85 \mathrm{~ms}$ [27]. Thus, a very accurate estimation of $\mathrm{T}_{1}$ values for a large range of $\mathrm{T}_{1}$ can be achieved. For example, it allows to detect in vitro Gd concentrations lower than $2.5 \mu \mathrm{M}$ (Figure 1D). From $\mathrm{T}_{1}$ maps acquired before and after Dotarem ${ }^{\circledR}$ injection, and knowing its $\mathrm{r}_{1}$ value at $7 \mathrm{~T}$ and $37^{\circ} \mathrm{C}$, one can estimate in vivo concentration maps as illustrated on Figure 1E, with the addition of a dedicated realignment procedure if needed. Finally, we verified that our quantification strategy follows a linear behavior with the injected dose, while the $\mathrm{T}_{1}$-weighted signal saturates at high concentrations as expected (Figure 1F).

The choice of spatial resolution is also key for a precise quantification of $D^{*}$. Indeed, low spatial resolution implies large partial volume effects leading to drastic errors in Gd 

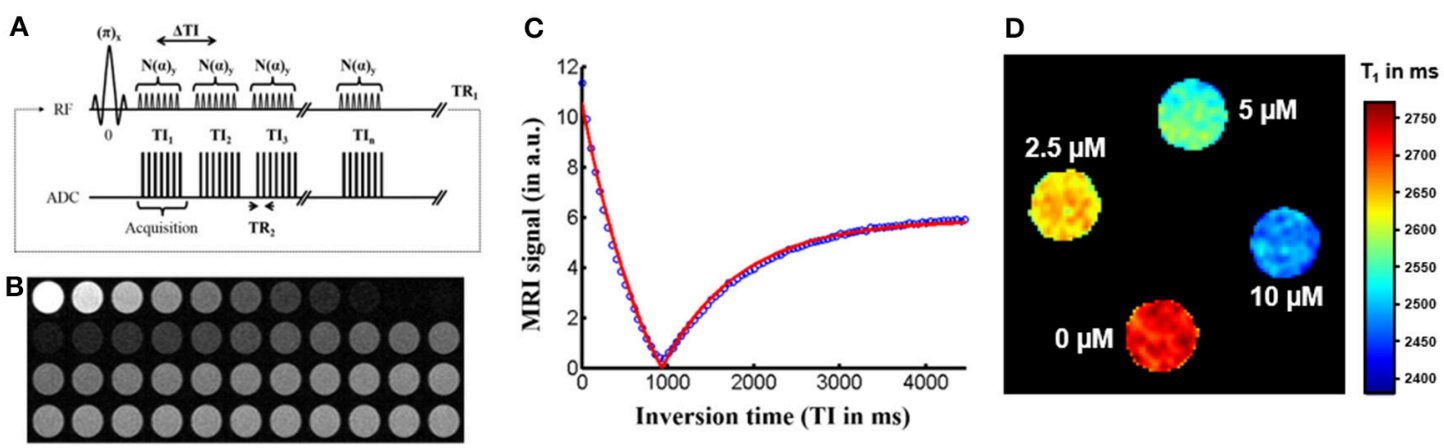

E
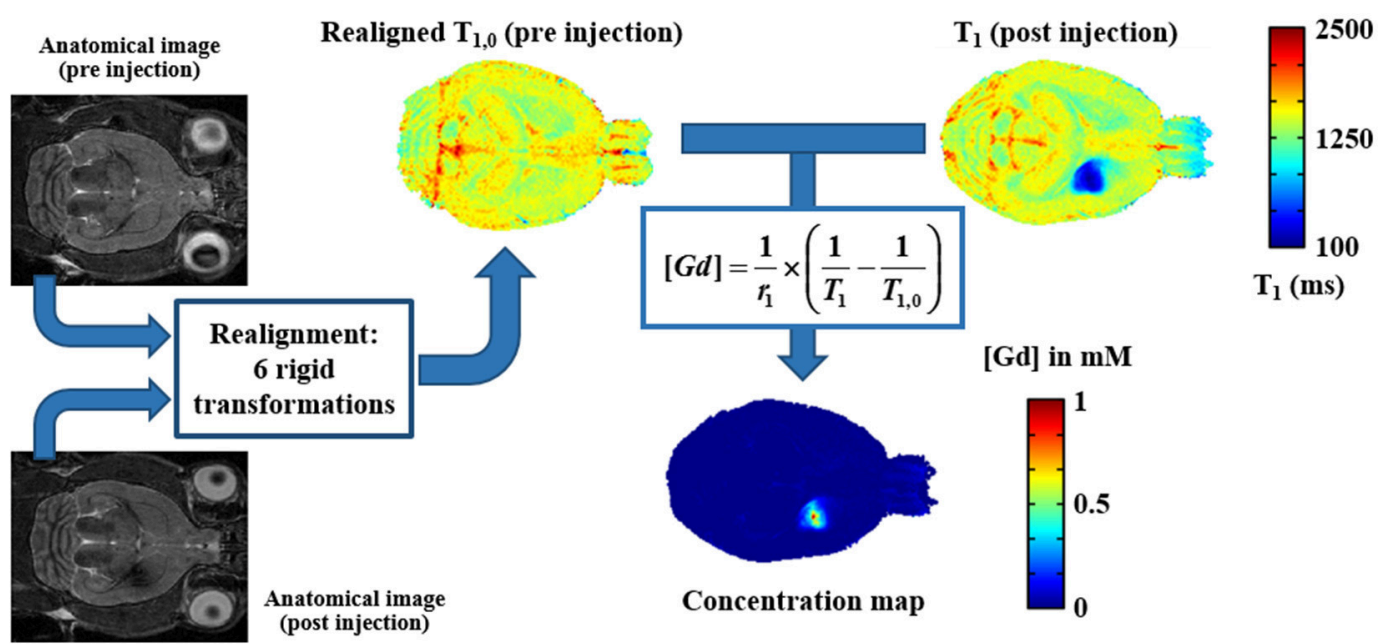

$\mathbf{F}$
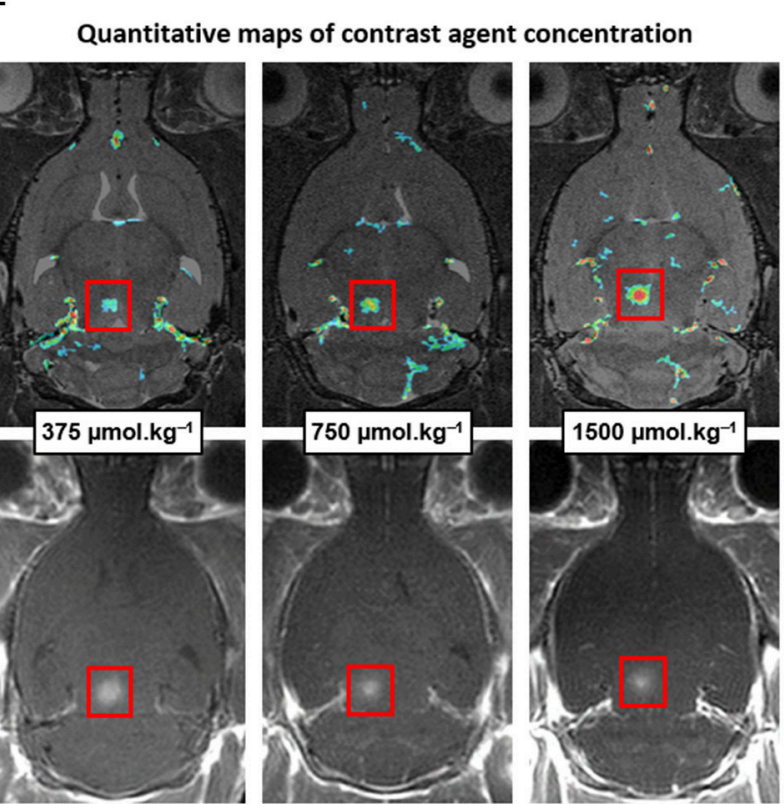

[Gd] in $\mathrm{mM}$

$\mathrm{T}_{1}$-weighted images
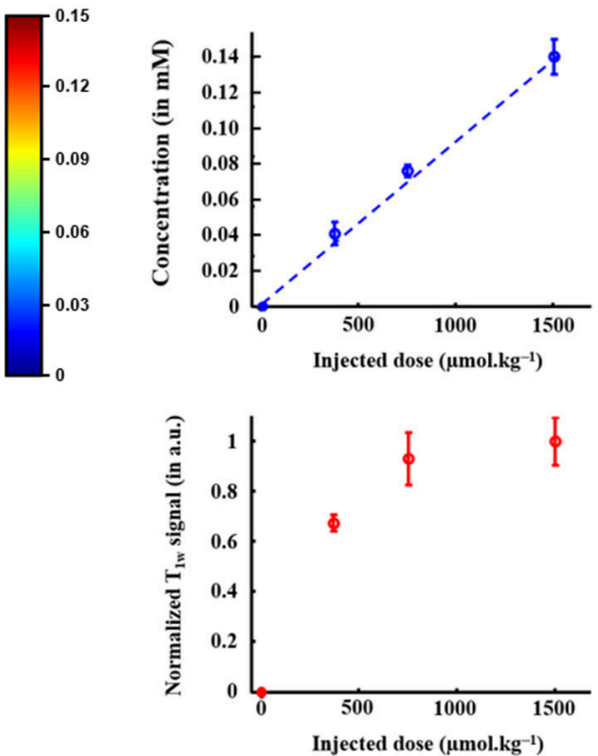

FIGURE 1 | MRI quantification of Gadolinium-based contrast agent concentration. (A) The $T_{1}$ mapping strategy is based on one Inversion Recovery Fast Gradient Echo sequence: it consists of a segmented series of fast gradient echo images (B) acquired at different time points after magnetization inversion. (C) The recovery of 
FIGURE 1 | the longitudinal magnetization is fitted voxel-by-voxel as a function of the inversion time to produce a quantitative $T_{1}$ map [31]. (D) The sensitivity of this $\mathrm{T}_{1}$ mapping strategy is estimated on a gallery of tubes filled with different concentrations of Dotarem ${ }^{\circledR}(0 / 2.5 / 5 / 10 \mu \mathrm{M})$. (E) From $\mathrm{T}_{1}$ parametric maps acquired before and after Gd-based contrast agent injection, and knowing the longitudinal relaxivity $r_{1}$ of the agent, one can estimate in vivo concentration map [32], with the addition of a realignment procedure if the animal is removed from the scanner between pre- and post-injection scans. (F) The concentration maps estimated with this $T_{1}$ mapping strategy are proportional to the injected dose, whereas the signal in standard $T_{1}$-weighted images saturates at high injected doses (Figure adapted from Marty et al. [33]: J Cereb Blood Flow Metab (2012) 32:1948-1958).

concentration mapping, while high resolution will result in poor signal-to-noise ratio and increased errors in $D^{*}$ estimation. In our case, Marty et al demonstrated from tortuosity values obtained by Thorne et al. [10] that an in-plane resolution of about $200 \mu \mathrm{m}$ was a good compromise given our sensitivity threshold. Slice thickness could be larger since our delivery method enables us to neglect the concentration gradient along depth (Figure 2D).

\section{FREE DIFFUSION COEFFICIENT MEASUREMENT}

The $D_{\text {free }}$ coefficient of Dotarem ${ }^{\circledR}$ was estimated by injecting a $5 \mu \mathrm{L}$ aliquot with a Hamilton syringe in a tube filled with dilute agar gel $(0.3 \% \mathrm{w} / \mathrm{w})$ maintained at $37^{\circ} \mathrm{C}$ (Figure 2A). According to Nicholson et al., this gel can be considered as an essentially free medium for diffusion [22]. The diffusion of Dotarem ${ }^{\circledR}$ was followed during $1 \mathrm{~h}$ by acquiring $\mathrm{T}_{1}$ maps as described above. A $\mathrm{T}_{10}$ map acquired before injection was used as a reference for estimating Gd concentration as follows [32]:

$$
[G d]=\frac{1}{r_{1}}\left(\frac{1}{T_{1}}-\frac{1}{T_{10}}\right)
$$

\section{COMPARISON OF TWO IN VIVO DELIVERY TECHNIQUES FOR TORTUOSITY ASSESSMENT IN RAT STRIATUM}

Dotarem ${ }^{\circledR}$ was delivered in vivo in the striatum of Sprague Dawley rats to estimate $D^{*}$. Two delivery protocols were compared. First, a direct intracranial injection was performed using a Hamilton syringe (Figure 2D). Second, a more complex but less invasive method was used (Figure 2E): an ultrasoundinduced $\mathrm{BBB}$ permeabilization protocol [33] was combined with an intravenous injection of Dotarem ${ }^{\circledR}$ to ensure a precise delivery where the ultrasound beam was focused [34, 35]. For both delivery methods, Dotarem ${ }^{\circledR}$ concentration maps were dynamically acquired for 1 hour as described before (Figures 2F,G).

\section{DATA PROCESSING}

Data analysis was performed using homemade Matlab routines (MathWorks, USA). MRI images were first reconstructed from raw K-space data, then $\mathrm{T}_{1}$ maps were obtained using the approach proposed in Deichmann et al. [31] and Deichmann and Haase [36]. 3D Gd concentration maps were calculated from the $\mathrm{T}_{1}$ maps acquired before and after injection (Figure 1E). On each slice of those maps and at every time point, the
Gd spatial distribution was fitted by a 2D Gaussian function (Figures 2B,F,G). As illustrated on Figures 2C,H,I, the diffusion coefficients along $\mathrm{X}$ and $\mathrm{Y}$ were computed as:

$$
D_{X}=\frac{\sigma_{X}^{2}}{2 t} \quad D_{Y}=\frac{\sigma_{Y}^{2}}{2 t}
$$

where $t$ is the acquisition time, $\sigma_{X}$ and $\sigma_{Y}$ are the Gaussian spreads along $X$ and $Y$ main axes. $D^{*}$ (resp. $D_{\text {free }}$ ) was taken as the average value along main axes obtained with in vivo (resp. in vitro) diffusion data.

\section{RESULTS}

The ECS tortuosity value obtained after direct intracerebral injection of Dotarem ${ }^{\circledR}$ was found equal to $3.25 \pm 0.40(n=2$ rats), while being equal to $1.70 \pm 0.11$ ( $n=3$ rats) if the probe diffuses within ECS after a local ultrasound-induced BBB permeabilization. The over-estimation of $\lambda$ value observed with the first delivery method probably comes from two major drawbacks of the intracerebral injection: (i) the tip diameter of the Hamilton syringe is rather large $(0.5 \mathrm{~mm})$ which is unfortunately expected to induce tissue inflammation along the needle pathway and thus locally increase the hindrance of molecular diffusion; (ii) $2 \mu \mathrm{L}$ of Dotarem ${ }^{\circledR}$ solution is injected as a bolus which induces significant changes of interstitial pressure at the injection site. For comparison, the RTI-TMA+ technique uses micropipettes and microelectrodes of $2-12 \mu \mathrm{m}$ diameter and nanoliters only are injected at slow speed.

On the other hand, the local ultrasound-induced BBB permeabilization does not modify the ECS diffusion properties. Indeed, the $\lambda$ values obtained with this delivery method are in good agreement with the ones obtained in the striatum of healthy brain with other techniques $(\lambda=1.59-1.60)[37,38]$.

\section{DISCUSSION AND CONCLUSION}

Characterization of the diffusion in the ECS is of great importance in order to predict drug biodistribution in the brain. Our view is that non-invasive imaging technique such as MRI and non-invasive probe delivery based on ultrasoundinduced $\mathrm{BBB}$ permeabilization can be combined to better probe brain tortuosity in vivo. Our ultrasound-based approach relies on the intravenous injection of the probe: its diffusion within brain tissue is thus very little disturbed as compared to a spontaneous crossing of the $\mathrm{BBB}$ through biochemical engineering.

Taking advantage of high field MRI acquisitions at 7T, we demonstrated that our $\mathrm{T}_{1}$ quantification strategy reaches 
A

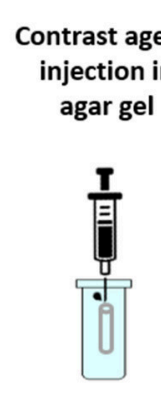

D

Contrast agent intracerebral injection

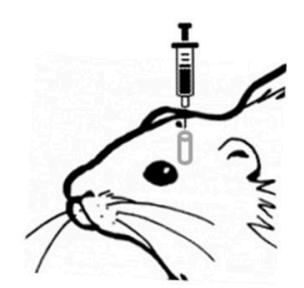

$\mathbf{F}$
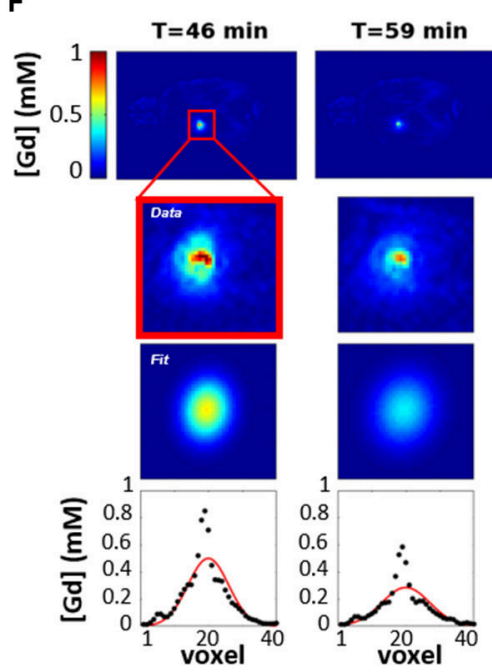

H

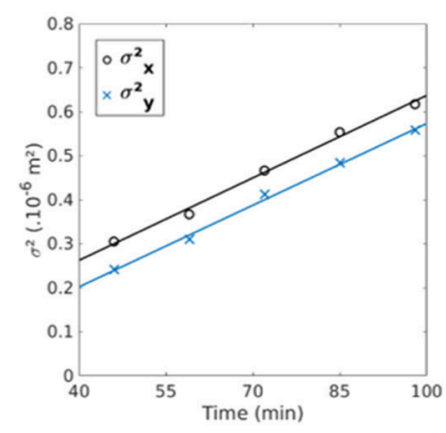

$\mathbf{E}$

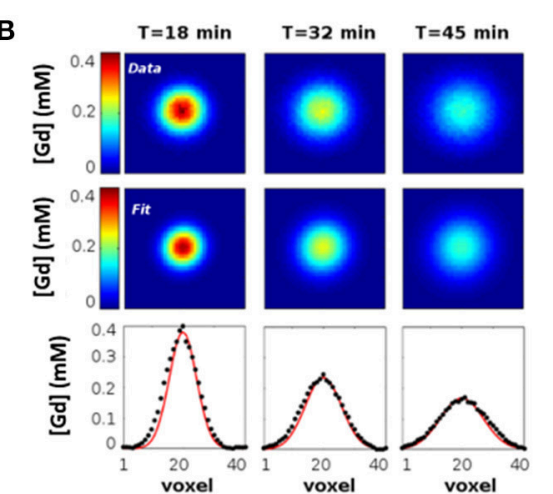

C

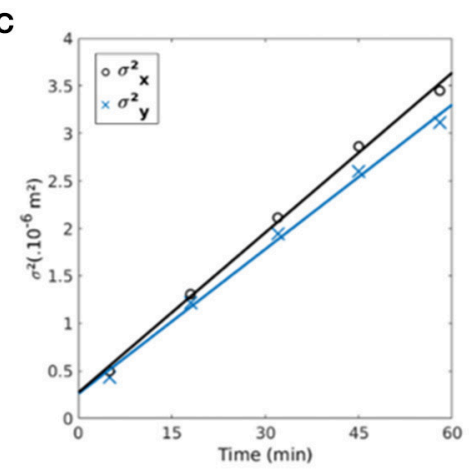

Contrast agent brain delivery with focused ultrasound

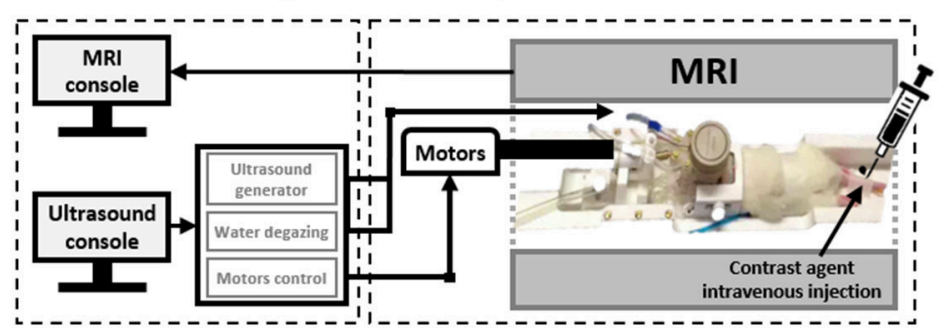

G

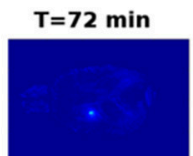

$\begin{array}{ll}\sum_{\text {E }} & 0.2 \\ \text { 导 } & 0.1 \\ & 0\end{array}$

$\mathrm{T}=15 \mathrm{~min}$
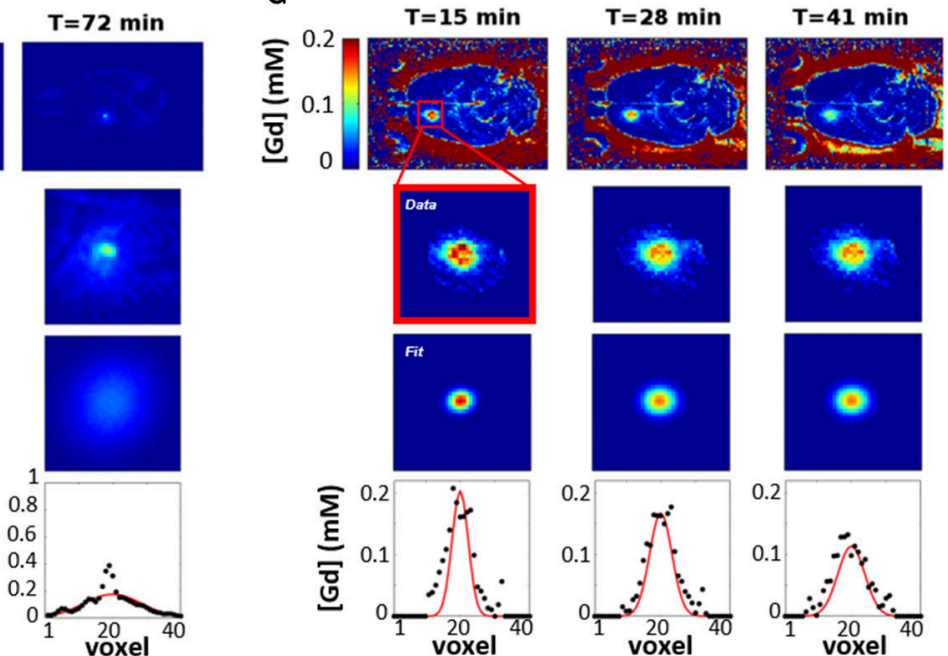

Fit

\section{c}
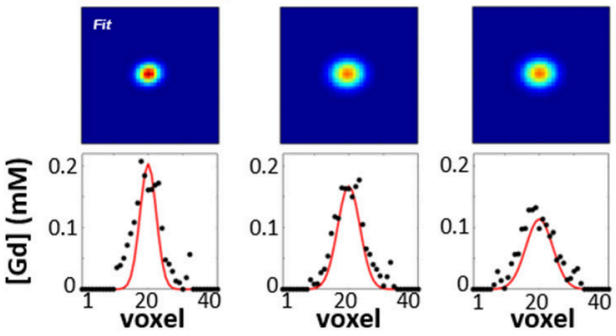

I

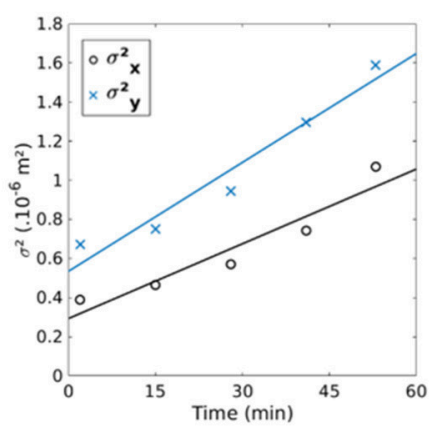

FIGURE 2 | MRI measurements of Gadolinium-based contrast agent diffusion. (A) The free diffusion coefficient $D_{\text {free }}$ of Dotarem ${ }^{\circledR}$ is estimated by injecting a $5 \mu \mathrm{L}$ aliquot with a Hamilton syringe in a tube filled with dilute agar gel $(0.3 \% \mathrm{~W} / \mathrm{W})$ maintained at $37^{\circ} \mathrm{C}$. (B) Concentration maps of Dotarem ${ }^{\circledR}$ (upper row) are acquired at 
FIGURE 2 | different time points after injection with the approach described in Figure 1. Each slice of those maps is fitted with a 2D Gaussian function (middle row). The result of this fit is also presented for the central line of the slice (lower row). (C) The squares of the $2 \mathrm{D}$ Gaussian spreads along $X$ and $Y$ main axes $\left(\sigma_{X}^{2}\right.$ and $\left.\sigma_{Y}^{2}\right)$ are fitted along time in order to estimate the diffusion coefficients $D_{X}$ and $D_{Y}$ : $D_{\text {free }}$ is taken as the average value of $D_{X}$ and $D_{Y}$. The effective diffusion coefficient $D^{\star}$ of Dotarem ${ }^{\circledR}$ is estimated in vivo in the striatum of Sprague Dawley rat for two delivery protocols: a direct intracranial injection of a $2 \mu \mathrm{L}$ bolus (D) and an ultrasound-induced BBB permeabilization protocol [34] combined with an intravenous injection (E). (F,G) For both protocols, concentration maps of Dotarem ${ }^{\circledR}$ (upper row) are acquired at different time points after injection with the approach described in Figure 1. Each slice of those maps is fitted with a 2D Gaussian function (middle row). The result of this fit is also presented for the central line of the slice (lower row). (H,I) For both protocols, the squares of the $2 \mathrm{D}$ Gaussian spreads along $X$ and $Y$ main axes $\left(\sigma_{X}^{2}\right.$ and $\left.\sigma_{Y}^{2}\right)$ are fitted along time in order to estimate the diffusion coefficients $D_{X}$ and $D_{Y}: D^{\star}$ is taken as the average value of $D_{X}$ and $D_{Y}$.

the requirements in terms of sensitivity detection, spatial and temporal resolutions, for estimating in vivo tortuosity values in deep regions of the rat brain. Our diffusion measurements are not based on diffusion-weighted MRI data that probe water diffusion at a few milliseconds timescale, but rather on the dynamic acquisition at a few minutes timescale of concentration maps of a Gd-based contrast agent diffusing in brain tissue. While optical methods study 2D diffusion processes occurring up to 2-3 min, and across distances up to about $300 \mu \mathrm{m}$, MRI can assess in $3 \mathrm{D}$ long range diffusion processes that evolve over hours across several millimeters. Both information can complement each other: a slower timescale should allow for example to investigate the cellular uptake and transport, as well as the potential clearance processes related to bulk flow or glymphatic pathway.

Our method presents the great advantages of not being restricted to superficial brain structures, of being compatible with a $3 \mathrm{D}$ anisotropic data analysis and of being usable multiple times on the same animal opening the door to longitudinal followup of tortuosity. This features are particularly relevant to know more about ECS structure in pathologies such as brain tumors, during aging, or under various pharmacological conditions. Indeed, other studies have already shown that this parameter can change in case of pathologies, such as ischemia and edema [17]. Furthermore, another interest of our approach is that additional MRI sequences can be added to the protocol for correlating tortuosity with structural, vascular, morphological, spectroscopic and functional MRI data. In a clinical context, the estimated values of tortuosity could be used as additional indicators of the pathological state.

Further interest in better ways to measure ECS physical properties has recently been raised by the discovery of major changes of ECS volume during sleep [15] and its correlation with the glymphatic pulsation flow that plays a great role in the cleaning of brain. Ultrafast MRI has recently been shown to be able to catch the slow flows occurring within the ECS [39]. In this context, such flow imaging could be combined with tortuosity measurements at various stages of the day/night cycle to better understand the clinical implications of the glymphatic pathway. Finally, Frenkel et al. have recently shown with histology that low intensity pulsed ultrasound could be used to transiently enlarge

\section{REFERENCES}

1. Syková E, Nicholson C. Diffusion in brain extracellular space. Physiol Rev. (2008) 88:1277-340. doi: 10.1152/physrev.00027.2007
ECS [40]. This could be transferred into clinical applications for facilitating drug access to targets, and the characterization of ECS diffusion properties with MRI would be of great importance to predict the drug biodistribution into the targeted area.

\section{ETHICS STATEMENT}

All in vivo experiments were conducted in strict accordance with the recommendations of the European Community (2010/63/EU) and the French legislation (decree no2013118) for use and care of laboratory animals. The protocol for contrast agent injection and $\mathrm{BBB}$ permeabilization has been approved by the Comité d'Éthique en Expérimentation Animale du Commissariat à l'Énergie Atomique et aux Énergies Alternatives - Direction des Sciences du Vivant Ile-de-France (CETEA/CEA/DSV IdF) under protocol ID 12-058.

\section{AUTHOR CONTRIBUTIONS}

SM developed and refined the perspective. SM, AC, and BL planned the MRI experiments. AC performed MRI acquisitions and data analysis. SM provided all data analysis pipelines. SM and BL managed the overall project and provided its funding. All authors contributed to manuscript edition.

\section{ACKNOWLEDGMENTS}

This work was part of the Iseult/INUMAC project, supported by the French public agency BPI (ex-OSEO), dedicated to the support of small and medium-sized companies.

France Life Imaging is acknowledged for its support in funding the NeuroSpin platform of preclinical MRI scanners.

AC received an Enhanced Eurotalents post-doctoral fellowship (Grant Agreement $\mathrm{n}^{\circ} 600382$ ), part of the Marie Sklodowska-Curie Actions Programme, co-funded by the European Commission and managed by the French Atomic Energy and Alternative Energies Commission (CEA).

The authors also thank the Health Technology Program of CEA for its support in funding some ultrasound experiments.

2. Nicholson C, Phillips JM. Ion diffusion modified by tortuosity and volume fraction in the extracellular microenvironment of the rat cerebellum. J Physiol. (1981) 321: 225-57. 
3. Ernst MH, Machta J, Dorfman JR, Vanbeijeren H. Long-time tails in stationary random-media. I. Theory. J Stat Phys (1984) 34:477-95. doi: 10.1007/BF01018555

4. Nicholson C. Diffusion and related transport mechanisms in brain tissue. Rep Prog Phys. (2001) 64:815-84. doi: 10.1088/0034-4885/6 $4 / 7 / 202$

5. Tao L, Nicholson C. Maximum geometrical hindrance to diffusion in brain extracellular space surrounding uniformly spaced convex cells. J Theor Biol. (2004) 229:59-68. doi: 10.1016/j.jtbi.2004.03.003

6. Chen KC, Nicholson C. Changes in brain cell shape create residual extracellular space volume and explain tortuosity behavior during osmotic challenge. Proc Natl Acad Sci USA. (2000) 97:8306-11. doi: $10.1073 /$ pnas. 150338197

7. Hrabětová $S$, Nicholson C. Contribution of dead-space microdomains to tortuosity of brain extracellular space. Neurochem Int. (2004) 45:467-77. doi: 10.1016/j.neuint.2003.11.011

8. Rusakov DA, Kullmann DM. Geometric and viscous components of the tortuosity of the extracellular space in the brain. Proc Natl Acad Sci USA. (1998) 95:8975-80.

9. Syková E. Extrasynaptic volume transmission and diffusion parameters of the extracellular space. Neuroscience (2004) 129:861-76. doi: 10.1016/j.neuroscience.2004.06.077

10. Thorne RG, Nicholson C. In vivo diffusion analysis with quantum dots and dextrans predicts the width of brain extracellular space. Proc Natl Acad Sci USA. (2006) 103:5567-72. doi: 10.1073/pnas.0509425103

11. Abbott NJ. Evidence for bulk flow of brain interstitial fluid: significance for physiology and pathology. Neurochem Int. (2004) 45:545-52. doi: 10.1016/j.neuint.2003.11.006

12. Iliff JJ, Wang M, Liao Y, Plogg BA, Peng W, Gundersen GA, et al. A paravascular pathway facilitates CSF flow through the brain parenchyma and the clearance of interstitial solutes, including amyloid $\beta$. Sci Transl Med. (2012) 4:147ra111. doi: 10.1126/scitranslmed.3003748

13. Aspelund A, Antila S, Proulx ST, Karlsen TV, Karaman S, Detmar M, et al. A dural lymphatic vascular system that drains brain interstitial fluid and macromolecules. J Exp Med. (2015) 212:991-9. doi: 10.1084/jem.20142290

14. Louveau A, Smirnov I, Keyes TJ, Eccles JD, Rouhani SJ, Peske JD, et al. Structural and functional features of central nervous system lymphatic vessels. Nature (2015) 523:337-41. doi: 10.1038/nature14432

15. Xie L, Kang H, Xu Q, Chen MJ, Liao Y, Thiyagarajan M, et al. Sleep drives metabolite clearance from the adult brain. Science (2013) 342:373-7. doi: $10.1126 /$ science. 1241224

16. Jin BJ, Smith AJ, Verkman AS. Spatial model of convective solute transport in brain extracellular space does not support a "glymphatic" mechanism. J Gen Physiol. (2016) 148:489-501. doi: 10.1085/jgp.201611684

17. Nicholson C, Syková E. Extracellular space structure revealed by diffusion analysis. Trends Neurosci (1998) 21:207-15. doi: 10.1016/S0166-2236(98)01261-2

18. Nicholson C, Kamali-Zare P, Tao L. Brain extracellular space as a diffusion barrier. Comput Vis Sci. (2011) 14:309-25. doi: 10.1007/s00791-012-0185-9

19. Nicholson C, Hrabětová S. Brain extracellular space: the final frontier of neuroscience. Biophys J (2017) 113:2133-42. doi: 10.1016/j.bpj.2017.06.052

20. Rall DP, Oppelt WW, Patlak CS. Extracellular space of brain as determined by diffusion of inulin from the ventricular system. Life Sci. (1962) 1:43-8. doi: 10.1016/0024-3205(62)90104-2

21. Nicholson C, Phillips JM. Ion diffusion modified by tortuosity and volume fraction in the extracellular microenvironment of the rat cerebellum. J Physiol. (1981) 321:225-57.

22. Nicholson C, Tao L. Hindered diffusion of high molecular weight compounds in brain extracellular microenvironment measured with integrative optical imaging. Biophys J. (1993) 65:2277-90. doi: 10.1016/S0006-3495(93)81324-9

23. Le Bihan D, Mangin JF, Poupon C, Clark CA, Pappata S, Molko N, et al. Diffusion tensor imaging: concepts and applications. J Magn Reson Imaging (2001) 13:534-46. doi: 10.1002/jmri.1076

24. Norris DG. The effects of microscopic tissue parameters on the diffusion weighted magnetic resonance imaging experiment. NMR Biomed. (2001) 14:77-93. doi: 10.1002/nbm.682

25. Kroenke CD, Ackerman JJ, Neil JJ. Magnetic resonance measurement of tetramethylammonium diffusion in rat brain: comparison of magnetic resonance and ionophoresis in vivo diffusion measurements. Magn Reson Med. (2003) 50:717-26. doi: 10.1002/mrm.10579

26. Duong TQ, Ackerman JJ, Ying HS, Neil JJ. Evaluation of extra- and intracellular apparent diffusion in normal and globally ischemic rat brain via 19F NMR. Magn Reson Med. (1998) 40:1-13. doi: 10.1002/mrm.1910400102

27. Marty B, Djemaï B, Robic C, Port M, Robert P, Valette J, et al. Hindered diffusion of MRI contrast agents in rat brain extracellular micro-environment assessed by acquisition of dynamic T1 and T2 maps. Contrast Media Mol Imaging (2013) 8:12-9. doi: 10.1002/cmmi.1489

28. Hagberg GE, Mamedov I, Power A, Beyerlein M, Merkle H, Kiselev VG, et al. Diffusion properties of conventional and calcium-sensitive MRI contrast agents in the rat cerebral cortex. Contrast Media Mol Imaging (2014) 9:71-82. doi: $10.1002 / \mathrm{cmmi} .1535$

29. Rogosnitzky M, Branch S. Gadolinium-based contrast agent toxicity: a review of known and proposed mechanisms. Biometals (2016) 29:365-76. doi: 10.1007/s10534-016-9931-7

30. Laurent S, Elst LV, Muller RN. Comparative study of the physicochemical properties of six clinical low molecular weight gadolinium contrast agents. Contrast Media Mol Imag. (2006) 1:128-37. doi: 10.1002/cmmi.100

31. Deichmann R, Hahn D, Haase A. Fast T1 mapping on a whole-body scanner. Magn Reson Med. (1999) 42:206-9. doi: 10.1002/(SICI)15222594(199907)42:1<206::AID-MRM28>3.0.CO;2-Q

32. Swift TJ, Connick RE. NMR-Relaxation mechanisms of $\mathrm{O} 17$ in aqueous solutions of paramagnetic cations and lifetime of water molecules in first coordination sphere. J Chem Phys. (1962) 37:307-20. doi: 10.1063/1.1701321

33. Marty B, Larrat B, Van Landeghem M, Robic C, Robert P, Port M, et al. Dynamic study of blood-brain barrier closure after its disruption using ultrasound: a quantitative analysis. J Cereb Blood Flow Metab. (2012) 32:194858. doi: 10.1038/jcbfm.2012.100

34. Magnin R, Rabusseau F, Salabartan F, Mériaux S, Aubry JF, Le Bihan D, et al. Magnetic resonance-guided motorized transcranial ultrasound system for blood-brain barrier permeabilization along arbitrary trajectories in rodents. $J$ Ther Ultrasound (2015) 3:22. doi: 10.1186/s40349-015-0044-5

35. Conti A, Magnin R, Gerstenmayer M, Lux F, Tillement O, Mériaux S, et al. Characterization of the diffusion process of different Gadoliniumbased nanoparticles within the brain tissue after ultrasound induced BloodBrain Barrier permeabilization. In: Ultrasonics Symposium (IUS), 2016 IEEE International, Tours.

36. Deichmann R, Haase A. Quantification of T1 values by SNAPSHOTFLASH NMR imaging. J Magn Reson. (1992) 96:608-12. doi: 10.1016/0022-2364(92)90347-A

37. Jansson A, Mazel T, Andbjer B, Rosén L, Guidolin D, Zoli M, et al. Effects of nitric oxide inhibition on the spread of biotinylated dextran and on extracellular space parameters in the neostriatum of the male rat. Neuroscience (1999) 91:69-80. doi: 10.1016/S0306-4522(98)00575-2

38. Reum T, Olshausen F, Mazel T, Vorísek I, Morgenstern R, Syková E. Diffusion parameters in the striatum of rats with 6-hydroxydopamine-induced lesions and with fetal mesencephalic grafts. J Neurosci Res (2002) 70:680-693. doi: 10.1002/jnr.10332

39. Kiviniemi V, Wang X, Korhonen V, Keinänen T, Tuovinen T, Autio J, et al. Ultra-fast magnetic resonance encephalography of physiological brain activity - Glymphatic pulsation mechanisms? J Cereb Blood Flow Metab (2016) 36:1033-45. doi: 10.1177/0271678X15622047

40. Frenkel V, Hersh DS, Anastasiadis P, Mohammadabadi A, Dancy J, Winkles J, et al. Pulsed focused ultrasound effects on the brain interstitium. In: Ultrasonics Symposium (IUS), 2017 IEEE International, Washington.

Conflict of Interest Statement: The authors declare that the research was conducted in the absence of any commercial or financial relationships that could be construed as a potential conflict of interest.

Copyright (c) 2018 Mériaux, Conti and Larrat. This is an open-access article distributed under the terms of the Creative Commons Attribution License (CC $B Y)$. The use, distribution or reproduction in other forums is permitted, provided the original author(s) and the copyright owner are credited and that the original publication in this journal is cited, in accordance with accepted academic practice. No use, distribution or reproduction is permitted which does not comply with these terms. 